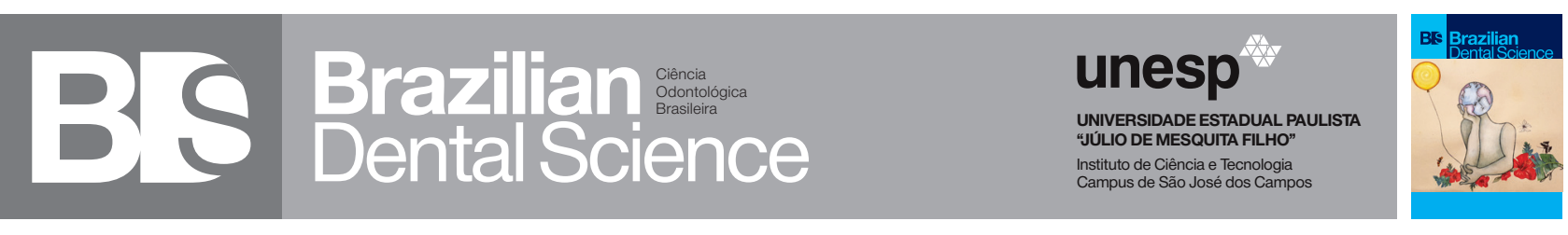

\title{
Anterior lingual mandibular bone cavity: a case report
}

Cavidade óssea mandibular lingual anterior: um relato de caso

Karen PINTADO-PALOMINO ${ }^{1}$, Camila TIRAPELLI ${ }^{2}$, Luciana Yamamoto ALMEIDA ${ }^{3}$, Jessica Luana dos SANTOS ${ }^{4}$, Lucas Ribeiro TEIXEIRA ${ }^{4}$, Emanuela Prado FERRAZ ${ }^{5}$, Samuel Porfirio XAVIER ${ }^{5}$, Jorge Esquiche LEÓN ${ }^{4}$

1 - Postgraduate Program in Oral Rehabilitation - Universidad Nacional Mayor de San Marcos (UNMSM) - Lima - Perú.

2 - Integrated Dental Clinic, Department of Dental Materials and Prosthodontics - School of Dentistry of Ribeirão Preto - University of São Paulo (FORP/USP) - Ribeirão Preto - SP - Brazil.

3 - Department of Clinical Medicine - Medical School of Ribeirão Preto - University of São Paulo (FMRP/USP).

4 - Oral Pathology, Department of Stomatology, Public Oral Health and Forensic Dentistry - School of Dentistry of Ribeirão Preto - University of São Paulo (FORP/USP) - Ribeirão Preto - SP - Brazil.

5 - Department of Oral and Maxillofacial Surgery and Periodontology - School of Dentistry of Ribeirão Preto - University of São Paulo (FORP/USP) - Ribeirão Preto - SP - Brazil.

\begin{abstract}
Stafne's bone cavity (SBC) is an asymptomatic lingual bone cavity situated near the angle of the mandible. The anterior variant of SBC, which shows a radiolucent unilateral ovoid lingual bone concavity in the canine-premolar mandibular region, is uncommon. A 73-year-old man was referred for assessment of loss of mandibular bone. Panoramic radiographs and computerized tomography scans showed a well-defined lingual bony defect in the anterior mandible. Analysis of imaginological documentation, made 14 years ago, revealed a progressive increase in mesiodistal diameter and intraosseous bony defect. The soft tissue obtained within the bony defect, microscopically revealed fibrous stroma containing blood vessels of varied caliber. The current anterior lingual mandibular bone defect case is probably caused by the salivary gland entrapped or pressure resorption, which can explain the SBC pathogenesis.
\end{abstract}

\section{KEYWORDS}

Bone defect; Mandible; Cone beam computed tomography; Diagnosis; Case report.

\section{RESUMO}

A cavidade óssea de Stafne (COS) é uma cavidade assintomática, localizada próximo ao ângulo da mandíbula, por lingual. A variante anterior da COS, a qual apresenta uma concavidade óssea lingual radiolúcida, ovoide e unilateral na região do caninopré-molar mandibular, é incomum. Um homem de 73 anos foi encaminhado para avaliação da perda óssea mandibular. A radiografia panorâmica e a tomografia computadorizada mostraram um defeito ósseo lingual bem definido na região anterior da mandibula. A análise da documentação imaginológica, realizada há 14 anos, revelou um aumento progressivo do diâmetro mesiodistal e defeito ósseo intraósseo. A biópsia do tecido mole obtido do defeito ósseo revelou microscópicamente estroma fibroso contendo vasos sanguíneos de calibre variado. O presente caso de defeito ósseo mandibular na região lingual anterior é provavelmente causado por glândula salivar aprisionada ou reabsorção por pressão, o que pode explicar a patogênese da COS.

\section{PALAVRAS-CHAVE}

Defeito ósseo; Madibula, tomografía de feixe cônico; Diagnostico; Relato de caso. 


\section{INTRODUCTION}

S tafne's bone cavity (SBC) is an uncommon anomaly (prevalence of $0.08 \%$ [1] - 0.7\% [2]) usually found in patients in the $5^{\text {th }}$ or $6^{\text {th }}$ decade of life and predominantly in males [1-4]. SBC has been described as an asymptomatic, well-defined radiolucent lingual bone cavity, mostly located in the posterior region of the mandible, below the inferior alveolar canal. The anterior variant of SBC, is uncommon [5], and often shows a radiolucent unilateral ovoid lingual bone concavity in the canine-premolar mandibular region, but bilateral variant has also been reported [6-8]. To the best of our knowledge, about 62 cases of SBC in the anterior mandible have been reported in the English-language literature (Table I). Moreover, unusual SBCs have been reported, including those cases with involvement of both lingual and buccal mandibular plates producing a tunnel-like lesion [9], location in the ramus of the mandible $[10,11]$, simultaneous unilateral anterior and posterior bony defect [12], atypical trilobate aspect [13] or lesions completely occupied by fatty tissue [14].

Etiopathogenesis of the SBC is not fully understood. Although not all compatible, embryological origin and vascular alterations have been proposed as a possible cause of SBC. Currently, bone resorption in response to pressure of salivary gland tissue is the most widely accepted hypothesis $[1,3,4,15]$, evidencing a peripheral origin [4]. Nevertheless, due to these defects are more frequently diagnosed in adults than children, it is suggested that the development of SBC probably occurs after the ossification of the mandible [3]. Interestingly, Philipsen et al. (2002) [3] suggest that a progressive reduction in bone volume could make that these depressions are visible on conventional radiographs around $35-40$ years of age.

The current case report describes an anterior lingual mandibular bone concavity (showing SBC features), with documentation over a period of 14 years. In addition, a list of anterior variant of SBC cases previously reported in the literature is presented.

\section{CASE REPORT}

A 73-year-old man was referred for assessing an asymptomatic anterior lingual mandibular bone defect. Medical history and extraoral examination were non-contributory. It was described a recent history of perforation of the lingual cortical bone, and whose interior was empty. He denied any history of trauma and bone surgical procedures in the lesional area. However, it is evident that tooth extractions were performed in areas close to the bony defect. Intraoral examination revealed a lingual mandibular bone cavity, which presented empty and covered with erythematous oral mucosa. The patient had no sensory or motor deficiency, and there was no pain or lymphadenopathy. Moreover, no periapical lesions of odontogenic origin, near bony defect were present. Repeated thermal pulp testing performed in non-endodontically treated teeth produced normal results. Cone beam computerized tomography (CBCT) showed on the right side of the mandible an ovoid-shaped hypodense lesion of approximately $1.2 \mathrm{~cm}$ (buccolingual width) $\times 3.8 \mathrm{~cm}$ (anteroposterior length), evidencing a mesiodistal diameter expansion, vestibular cortical thinning and lingual cortical bone destruction (Figure 1). Histopathological examination of the soft tissue obtained within the bony defect was performed, which revealed fibrous stroma containing blood vessels of varied caliber (Figure 2). The clinicopathological correlation favored a diagnosis of variant anterior of SBC. Interestingly, two panoramic radiographs, taken in 2002 and 2010, as well as CBCT taken in 2010, could be evaluated after review of patient's record (Figure 3 and 4). Noteworthy, they showed a progressive unilocular radiolucent area located in the anterior region of the right mandible below the apices of the second premolar (tooth \# 29) to the canine (tooth \# 27). Additionally, root canal treated teeth (tooth \# 29 and tooth \# 28) were observed (Figure 3a) and subsequently the loss of tooth \# 29. Notice at level of tooth \# 28 a discreet widening of the apical periodontium without contact with the radiolucent area (Figure 3b). In fact, both panoramic radiographs (Figure 3), show that the radiolucent area was distant and unrelated to the tooth apices, and by comparative analysis, there was a progressive increase in mesiodistal diameter of the radiolucent area. The CBCT scans performed in 2010 showed a well-defined lingual bony defect in the anterior mandible with expansion of the lingual cortical bone (Figure 4). 

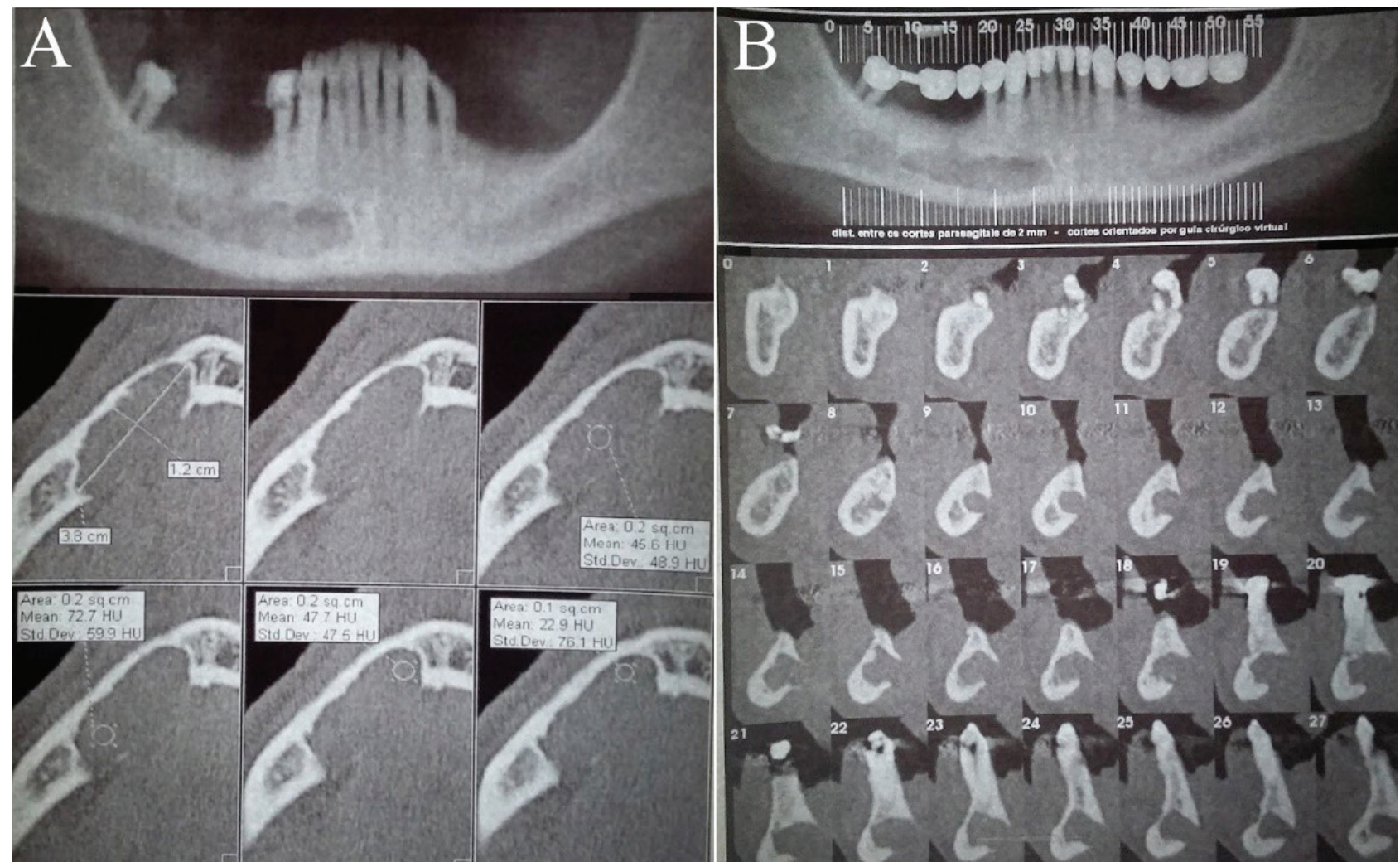

Figure 1 - Cone beam computerized tomography (CBCT) taken in 2015, showing an unilateral ovoid-shaped hypodense lesion with increased dimensions. Axial (a) and cross-sectional (b) views show the complete destruction of the lingual cortical bone.

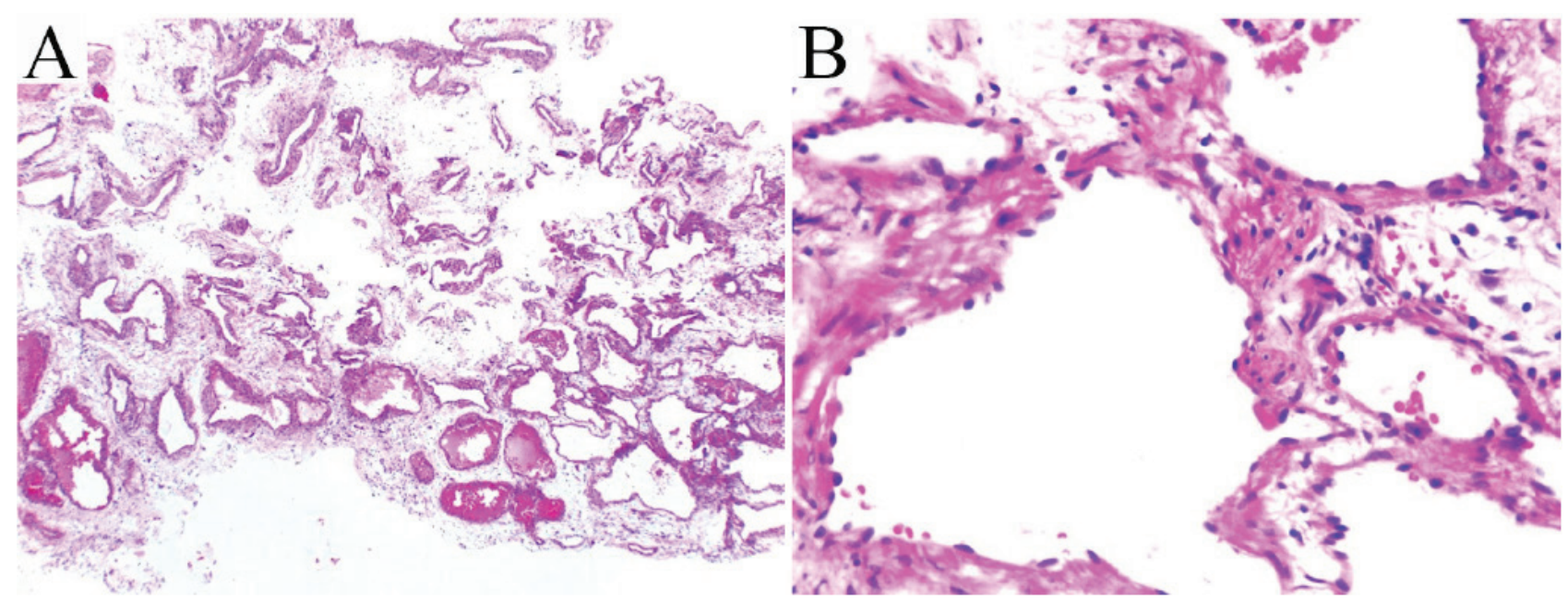

Figure 2 - Microscopic examination of soft tissue lining obtained from the lingual bony defect, revealed numerous blood vessels of varying size, supported by loose connective tissue (a, x4; b, x40; H\&E stain). 

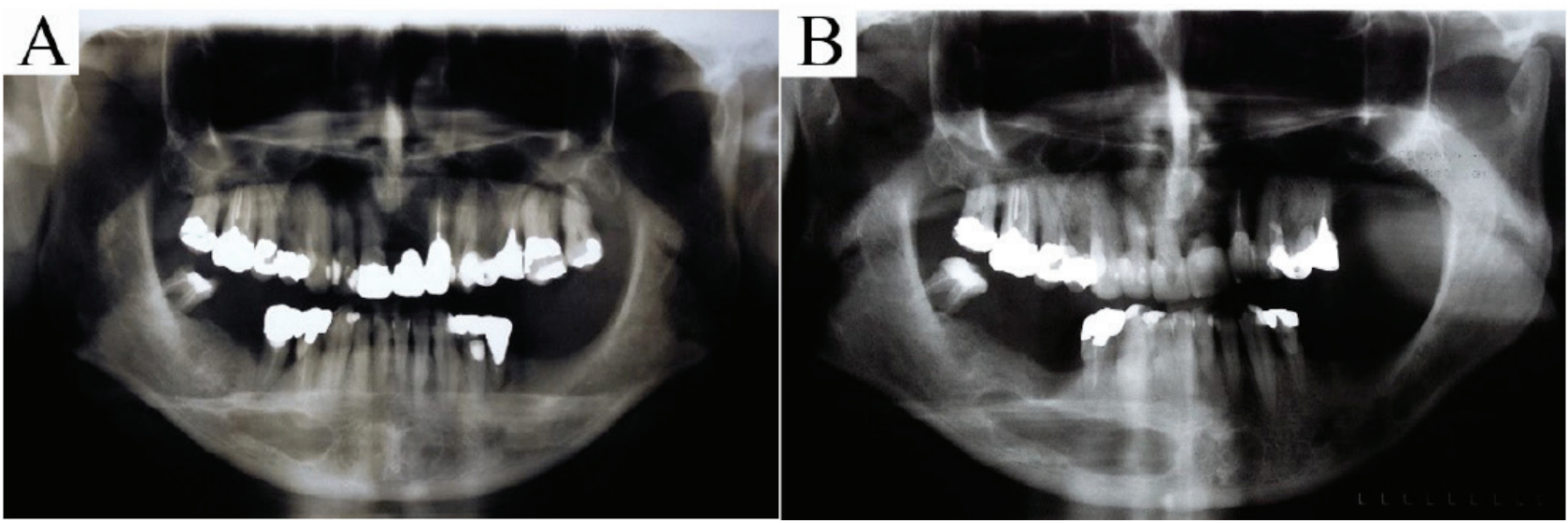

Figure 3 - Panoramic radiography images revealing a unilateral bone defect in the anterior mandible. (a) Image taken in 2002 , revealing a unilocular radiolucent lesion in the canine and premolar area of the right mandible. (b) Image taken in 2010, showing a progressive increase of the lesion in mesiodistal diameter.
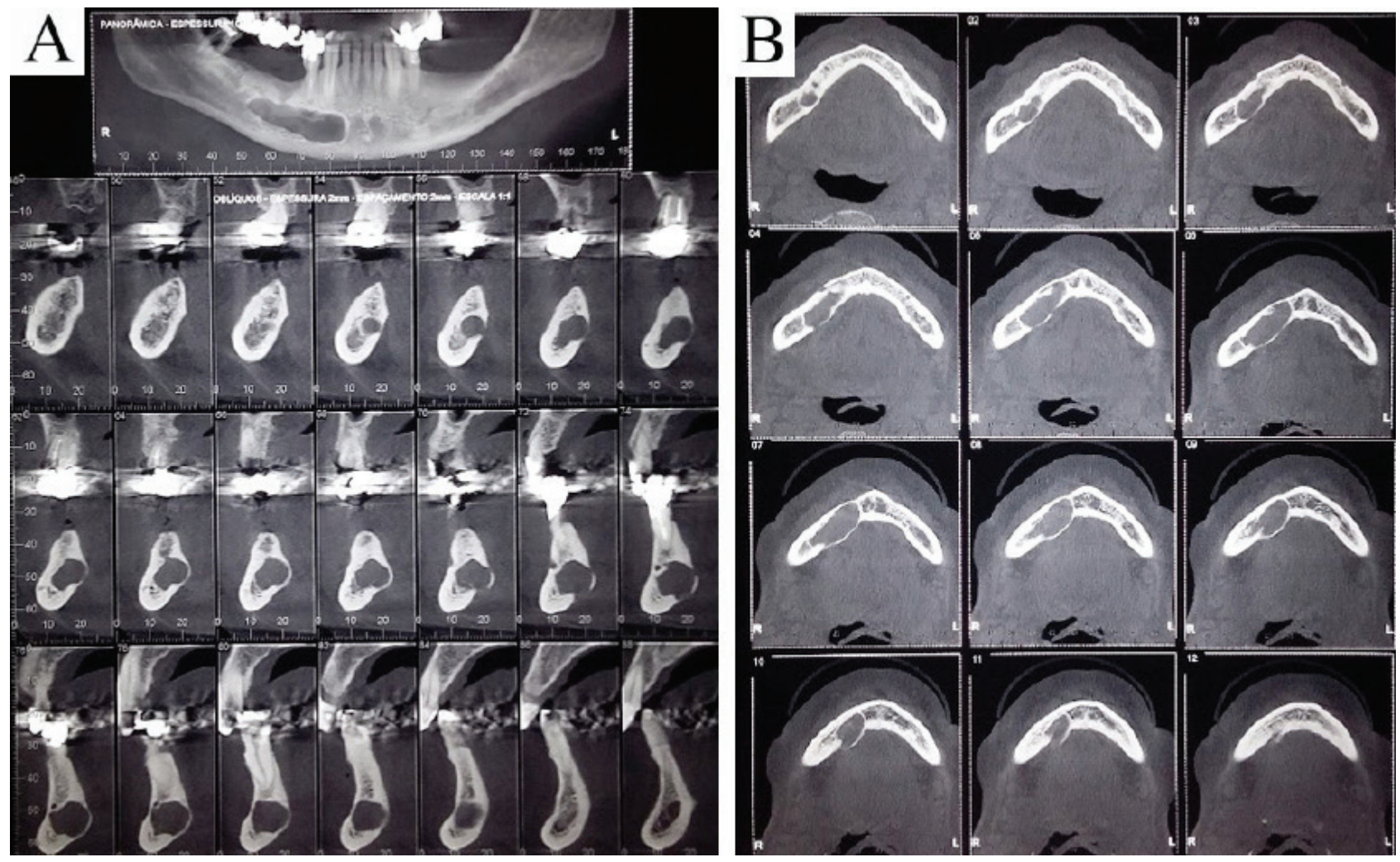

Figure 4 - Cone beam computerized tomography (CBCT) taken in 2010, showing unilateral ovoid-shaped hypodense lesion expanding the lingual cortical bone, in cross-sectional (a) and axial (b) views. 


\section{DISCUSSION}

Based on studies of contemporary and archaeological cases, Philipsen (2002) [3] has described four topographical variants of SBC: 1) located in lingual anterior mandibular body (incisor-canine-premolar area), above the mylohyoid muscle; 2) located posterior to the mandibular angle-first permanent molar area, below the mandibular canal; 3) located to the ascending, lingual mandibular ramus, posterior to the lingual foramen, below the neck of the condyle; and 4) located to the buccal aspects of the ascending mandibular ramus (extremely rare). In general, posterior variant of SBC occurs most frequently [2$4,15]$ showing an prevalence of approximately $0.081 \%$ while the prevalence in anterior variant is $0.003 \%$ [16].

The review (English language literature) shown in the Table 1 , presents a clinical data based on 63 cases of lingual bone cavity in the anterior mandible (including the current case) between 1957 and 2019. According to the anterior variant of mostly affected male patients (76\%) with age ranging from 18 [17] to 76 years [12] (mean age, 46 years). This variant is most frequently described as an oval radiolucent unilateral lesion. Radiographically, the anterior variant presents less frequently well-defined sclerotic borders than posterior variant [3]. This finding could also explain why the diagnosis of anterior variant of SBC not considered in the differential diagnosis.

Regarding the location of the anterior variant of SBC, both sides of the mandible with similar frequency are affected, and mainly the premolar - canine region. Less common, this variant can have bilateral presentation ( 07 cases in men and 04 cases in women), appearing as a radiolucent lesion in each side of the mandible $[7,8,18,19]$ or as a single radiolucent lesion crossing the midline [20-22] that may extend in the whole body of the mandible $[6,17,23]$. Additionally, Ozaki et al. (2015) [12] have reported a SBC which presented simultaneously anterior and posterior unilateral location.
The pathogenesis of the SBC is still controversial. Originally, the embryologic theory [24] has argued that a mandibular ossification defect of Meckel's cartilage could be the cause of this entity. Likewise, an entrapment of salivary gland tissue during ossification of the mandible has been suggested [10]. However, these theories are being disregarded because SBC is observed mostly in patients aged over 40 years [4].

On the other hand, most authors have accepted the pressure resorption theory $[1,3,4,15]$ and considered it as the main etiological factor for majority of cases [25]. According to this theory, due to a chronic inflammatory process, the salivary gland hypertrophy is capable of exerting enough pressure to cause bone resorption. Apparently, there would be a relationship between the bone cavity and its corresponding submandibular gland $[3,25]$. Thus, hypertrophic submandibular gland could be the cause of the posterior variant of SBC, whereas an aberrant anterior lobe of the sublingual gland could be the cause of the anterior variant of SBC [4]. The lymphocytic infiltration found in salivary gland tissue, as possible cause of hypertrophied salivary glands, has been suggested to corroborate the theory [8] reinforcing the peripheral origin [4]. However, in contrast to this theory, some SBC case reports have revealed the absence of salivary gland tissue within the bony defect [26], whereas other studies showed presence of adipose tissue and bony fragments inside the bone cavity $[14,27,28]$. Interestingly, only 2 cases of the anterior variant of SBC have been reported presenting each one of them, among others, connective tissue (case 4) or blood vessels (case 62) (Table 1). In the current case, fibrous stroma containing blood vessels of varied caliber was observed. It should be noted that the tissue obtained was part of the lining of an empty central cavity, highly consistent with a reactive nature, and different from a vascular malformation or neoplasia. 
The most SBC cases have been described as an asymptomatic and non-progressive anomaly [5, 29, 30]. Therefore, non-invasive diagnosis techniques have been preconized, avoiding surgical interventions, which could be an unnecessary option in the management of SBC [31]. In the current case, additional histological examination was required for accurate diagnosis, due to their progressive growing pattern, observed during the patient's follow-up.

The SBC usually deserves no treatment $[14,32]$, but when performed, conservative treatment is recommended [6]. Management should be based on integrated clinical and imaginological approach $[9,33]$ or even three-dimensional imaging to rule out other odontogenic and non-odontogenic lesions [34].

To the best of our knowledge, about 62 cases of SBC in the anterior mandible have been reported in the English-language literature (Table I).

Table I - "Stafne bone cavity cases in the anterior mandible"

\begin{tabular}{|c|c|c|c|c|c|}
\hline Case & Authors (Year) & $\begin{array}{l}\text { Age/ } \\
\text { Sex }\end{array}$ & $\begin{array}{l}\text { Unilateral/ } \\
\text { Bilateral }\end{array}$ & Site & Content \\
\hline 1 & $\begin{array}{c}\text { Richard and } \\
\text { Ziskind (1957) } \\
\text { [35] }\end{array}$ & $46 / M$ & Unilateral & $\begin{array}{l}\text { Left canine and left } \\
\text { first premolar }\end{array}$ & SG \\
\hline 2 & $\begin{array}{c}\text { Araiche and } \\
\text { Brode (1959) [36] }\end{array}$ & $55 / M$ & Unilateral & $\begin{array}{l}\text { Right central incisor } \\
\text { to right premolar } \\
\text { (edentulous) }\end{array}$ & SG \\
\hline 3 & $\begin{array}{c}\text { Camilleri(1963) } \\
\text { [20] }\end{array}$ & $31 / F$ & Bilateral & $\begin{array}{l}\text { Incisal region } \\
\text { (edentulous) }\end{array}$ & SG \\
\hline 4 & $\begin{array}{c}\text { Bergenholtz and } \\
\text { Persson (1963) } \\
\text { [27] }\end{array}$ & $50 / \mathrm{M}$ & Unilateral & $\begin{array}{l}\text { Left canine and left } \\
\text { first premolar }\end{array}$ & $\mathrm{CT}, \mathrm{BF}$ \\
\hline 5 & $\begin{array}{c}\text { Friedman }(1964) \\
{[21]}\end{array}$ & $54 / M$ & Bilateral & $\begin{array}{l}\text { Right canine to left } \\
\text { canine }\end{array}$ & $\mathrm{SG}$ \\
\hline 6 & $\begin{array}{l}\text { Palladino et al. } \\
\text { (1965) [37] }\end{array}$ & $40 / \mathrm{M}$ & Unilateral & $\begin{array}{l}\text { Right canine and } \\
\text { right first premolar }\end{array}$ & SG \\
\hline 7 & $\begin{array}{c}\text { Abramson (1966) } \\
{[38]}\end{array}$ & $26 / F$ & Unilateral & $\begin{array}{l}\text { Right canine } \\
\text { (edentulous) }\end{array}$ & SG \\
\hline 8 & $\begin{array}{l}\text { Miller and Winni- } \\
\text { ck (1971) [22] }\end{array}$ & $48 / M$ & Bilateral & $\begin{array}{l}\text { Between central } \\
\text { incisors }\end{array}$ & SG \\
\hline 9 & $\begin{array}{l}\text { Malkin and Berg } \\
\text { (1974) [39] }\end{array}$ & $54 / M$ & Bilateral & $\begin{array}{l}\text { Right canine to left } \\
\text { canine }\end{array}$ & SG \\
\hline 10 & $\begin{array}{c}\text { Forrest (1974) } \\
{[40]}\end{array}$ & $\begin{array}{l}\text { Lateral } \\
\text { incisor } \\
\text { and } \\
\text { canine }\end{array}$ & SG & $\begin{array}{l}\text { Right canine and } \\
\text { right first premolar }\end{array}$ & SG \\
\hline 11 & $\begin{array}{l}\text { Stene and Peder- } \\
\text { sen (1977) [41] }\end{array}$ & $\begin{array}{l}\text { Right } \\
\text { canine }\end{array}$ & SG & $\begin{array}{l}\text { Right canine } \\
\text { (edentulous) }\end{array}$ & SG \\
\hline 12 & Plezia (1977) [42] & $31 / \mathrm{M}$ & Unilateral & Right premolars & SG \\
\hline
\end{tabular}

\begin{tabular}{|c|c|c|c|c|c|}
\hline Case & Authors(Year) & $\begin{array}{l}\text { Age/ } \\
\text { Sex }\end{array}$ & $\begin{array}{c}\text { Unilateral/ } \\
\text { Bilateral }\end{array}$ & Site & Content \\
\hline 12 & Plezia (1977) [42] & $31 / \mathrm{M}$ & Unilateral & Right premolars & SG \\
\hline 13 & $\begin{array}{c}\text { Connor (1979) } \\
\text { [26] }\end{array}$ & $56 / \mathrm{M}$ & Unilateral & Left canine & Empty \\
\hline 14 & $\begin{array}{c}\text { Sandy and } \\
\text { Williams (1981) } \\
\text { [43] }\end{array}$ & $53 / \mathrm{M}$ & Unilateral & Right lateral incisor & SG \\
\hline 15 & $\begin{array}{c}\text { Layne etal. (1981) } \\
\text { [44] }\end{array}$ & $20 / F$ & Unilateral & $\begin{array}{l}\text { Right lateral incisor } \\
\text { to first premolar } \\
\text { (edentulous) }\end{array}$ & SG \\
\hline 16 & $\begin{array}{l}\text { Gosney and Giles } \\
\text { (1981) [45] }\end{array}$ & $20 / M$ & Unilateral & $\begin{array}{l}\text { Right second premo- } \\
\text { lar and right first } \\
\text { molar (edentulous) }\end{array}$ & SG \\
\hline 17 & $\begin{array}{l}\text { Mizuno et al. } \\
\text { (1983) [23] }\end{array}$ & $36 / \mathrm{M}$ & Bilateral & $\begin{array}{l}\text { From right first } \\
\text { molar to left second } \\
\text { premolar }\end{array}$ & SG \\
\hline 18 & $\begin{array}{l}\text { Hayashi et al. } \\
\text { (1984) [46] }\end{array}$ & $46 / \mathrm{M}$ & Unilateral & $\begin{array}{l}\text { Left central incisor } \\
\text { and left lateral } \\
\text { incisor }\end{array}$ & SG \\
\hline 19 & $\begin{array}{l}\text { Miller and Fanta- } \\
\text { sia (1986) [47] }\end{array}$ & $27 / M$ & Unilateral & $\begin{array}{l}\text { Between second } \\
\text { premolar and first } \\
\text { molar }\end{array}$ & SG \\
\hline 20 & $\begin{array}{c}\text { Ström and } \\
\text { Fjellström (1987) } \\
\text { [48] }\end{array}$ & $61 / M$ & Unilateral & Left canine & SG \\
\hline 21 & $\begin{array}{l}\text { Anneroth et al. } \\
\text { (1990) [49] }\end{array}$ & $57 / M$ & Unilateral & $\begin{array}{l}\text { Between left lateral } \\
\text { incisor and left } \\
\text { canine }\end{array}$ & SG \\
\hline 22 & $\begin{array}{l}\text { Tominaga et al. } \\
\text { (1990) [50] }\end{array}$ & $54 / \mathrm{M}$ & Unilateral & $\begin{array}{l}\text { Right canine and } \\
\text { right premolars }\end{array}$ & SG \\
\hline 23 & $\begin{array}{l}\text { Grellner et al. } \\
\text { (1990) [17] }\end{array}$ & $18 / M$ & Bilateral & $\begin{array}{l}\text { From right second } \\
\text { premolar to left } \\
\text { second premolar }\end{array}$ & SG \\
\hline 24 & $\begin{array}{c}\text { Grellner et al. } \\
\text { (1990) [17] }\end{array}$ & $24 / M$ & Bilateral & $\begin{array}{l}\text { Lateral incisor and } \\
\text { first premolar }\end{array}$ & No biopsy \\
\hline 25 & $\begin{array}{l}\text { Childers et al. } \\
\text { (1990) [51] }\end{array}$ & $26 / \mathrm{M}$ & Unilateral & Right central incisor & SG \\
\hline 26 & $\begin{array}{l}\text { Buchner etal. } \\
\text { (1991) [28] }\end{array}$ & $39 / \mathrm{M}$ & Unilateral & Canine & SG \\
\hline 27 & $\begin{array}{l}\text { Buchner et al. } \\
\text { (1991) [28] }\end{array}$ & $50 / \mathrm{M}$ & Unilateral & Canine & AT \\
\hline 28 & $\begin{array}{l}\text { Buchner et al. } \\
\text { (1991) [28] }\end{array}$ & $64 / \mathrm{M}$ & Unilateral & Canine & SG \\
\hline 29 & $\begin{array}{l}\text { Buchner et al. } \\
\text { (1991) [28] }\end{array}$ & $51 / \mathrm{M}$ & Unilateral & Incisors & SG \\
\hline 30 & $\begin{array}{c}\text { Salman and } \\
\text { Chaudhry (1991) } \\
\text { [52] }\end{array}$ & $25 / F$ & Unilateral & Incisors & SG \\
\hline 31 & $\begin{array}{l}\text { Barak etal. } \\
\text { (1993) [53] }\end{array}$ & $46 / \mathrm{M}$ & Unilateral & Left premolars & SG \\
\hline 32 & $\begin{array}{l}\text { Thomas and } \\
\text { Abramovitch } \\
\text { (1996) [54] }\end{array}$ & $26 / F$ & Unilateral & Left canine & - \\
\hline 33 & $\begin{array}{c}\text { Apruzzese and } \\
\text { Longoni (1999) } \\
\text { [55] }\end{array}$ & $45 / F$ & Unilateral & Left first premolar & SG \\
\hline 34 & $\begin{array}{c}\text { Katz etal.(2001) } \\
\text { [56] }\end{array}$ & $49 / \mathrm{M}$ & Unilateral & Second left premolar & No biopsy \\
\hline 35 & $\begin{array}{l}\text { de Courten et al. } \\
\text { (2002) [57] }\end{array}$ & $42 / M$ & Unilateral & $\begin{array}{l}\text { Between left first } \\
\text { premolar and left } \\
\text { second molar } \\
\text { (edentulous) }\end{array}$ & SG \\
\hline
\end{tabular}




\begin{tabular}{|c|c|c|c|c|c|}
\hline Case & Authors(Year) & $\begin{array}{l}\text { Agel } \\
\text { Sex }\end{array}$ & $\begin{array}{l}\text { Unilateral/ } \\
\text { Bilateral }\end{array}$ & Site & Content \\
\hline 35 & $\begin{array}{l}\text { de Courten et al. } \\
\text { (2002) [57] }\end{array}$ & $42 / \mathrm{M}$ & Unilateral & $\begin{array}{l}\text { Between left first } \\
\text { premolar and left } \\
\text { second molar } \\
\text { (edentulous) }\end{array}$ & SG \\
\hline 36 & $\begin{array}{c}\text { Dorman and } \\
\text { Pierse (2002) } \\
{[58]}\end{array}$ & $45 / F$ & Unilateral & $\begin{array}{l}\text { Between right } \\
\text { central incisor and } \\
\text { right first premolar }\end{array}$ & SG \\
\hline 37 & $\begin{array}{l}\text { Philipsen et al. } \\
\text { (2002) [3] }\end{array}$ & NM & NM & $\begin{array}{l}\text { Incisors, canine, } \\
\text { premolars }\end{array}$ & No biopsy \\
\hline 38 & $\begin{array}{l}\text { Philipsen et al. } \\
\text { (2002) [3] }\end{array}$ & NM & NM & $\begin{array}{l}\text { Incisors, canine, } \\
\text { premolars }\end{array}$ & No biopsy \\
\hline 39 & $\begin{array}{l}\text { Philipsen et al. } \\
\text { (2002) [3] }\end{array}$ & NM & NM & $\begin{array}{l}\text { Incisors, canine, } \\
\text { premolars }\end{array}$ & No biopsy \\
\hline 40 & $\begin{array}{l}\text { Philipsen et al. } \\
\text { (2002) [3] }\end{array}$ & NM & NM & $\begin{array}{l}\text { Incisors, canine, } \\
\text { premolars }\end{array}$ & No biopsy \\
\hline 41 & $\begin{array}{l}\text { Phillips and Yates } \\
\text { (2004) [59] }\end{array}$ & $52 / \mathrm{M}$ & Unilateral & Right first premolar & No biopsy \\
\hline 42 & $\begin{array}{l}\text { Queiroz et al. } \\
(2004)[18]\end{array}$ & $32 / F$ & Bilateral & $\begin{array}{l}\text { Right lateral incisor } \\
\text { - canine and left } \\
\text { lateral incisor-canine }\end{array}$ & $S G$ \\
\hline 43 & $\begin{array}{l}\text { Belmonte-Caro } \\
\text { et al. (2005) [60] }\end{array}$ & $68 / \mathrm{M}$ & Unilateral & $\begin{array}{l}\text { Left canine and left } \\
\text { first premolar }\end{array}$ & SG \\
\hline 44 & $\begin{array}{l}\text { Solomon et al. } \\
\text { (2006) [61] }\end{array}$ & $55 / F$ & Unilateral & Canine & No biopsy \\
\hline 45 & $\begin{array}{l}\text { Herbozo Silva et } \\
\text { al. (2006) [6] }\end{array}$ & $33 / M$ & Bilateral & $\begin{array}{c}\text { From right first } \\
\text { molar to left first } \\
\text { molar }\end{array}$ & SG \\
\hline 46 & $\begin{array}{c}\text { Nah etal. (2007) } \\
{[62]}\end{array}$ & $47 / M$ & Unilateral & Left premolars & SG \\
\hline 47 & $\begin{array}{l}\text { Smith et al. } \\
(2007)[7]\end{array}$ & $25 / F$ & Bilateral & $\begin{array}{l}\text { Canine and pre- } \\
\text { molars }\end{array}$ & No biopsy \\
\hline 48 & $\begin{array}{l}\text { Bornstein et al. } \\
\text { (2009) [63] }\end{array}$ & $47 / M$ & Unilateral & $\begin{array}{l}\text { Right canine to right } \\
\text { second premolar }\end{array}$ & No biopsy \\
\hline 49 & $\begin{array}{l}\text { Bornstein et al. } \\
\text { (2009) [63] }\end{array}$ & $62 / M$ & Unilateral & $\begin{array}{l}\text { Left lateral incisor } \\
\text { and left canine }\end{array}$ & No biopsy \\
\hline 50 & $\begin{array}{l}\text { Turkoglu and } \\
\text { Orhan (2010) [5] }\end{array}$ & $52 / \mathrm{M}$ & Unilateral & Right canine & SG \\
\hline 51 & $\begin{array}{l}\text { Florin and Man- } \\
\text { del(2010) [64] }\end{array}$ & $68 / \mathrm{M}$ & Unilateral & $\begin{array}{c}\text { Right lateral incisor } \\
\text { to right first pre- } \\
\text { molar }\end{array}$ & No biopsy \\
\hline 52 & $\begin{array}{l}\text { Sisman etal. } \\
(2010)[16]\end{array}$ & $62 / F$ & Unilateral & $\begin{array}{l}\text { Right canine and } \\
\text { right premolar } \\
\text { (edentulous) }\end{array}$ & No biopsy \\
\hline 53 & $\begin{array}{c}\text { Krafft et al. (2010) } \\
{[33]}\end{array}$ & $46 / \mathrm{M}$ & Unilateral & $\begin{array}{l}\text { Left lateral incisor to } \\
\text { left first premolar }\end{array}$ & SG \\
\hline 54 & $\begin{array}{c}\text { Voss etal. (2010) } \\
{[65]}\end{array}$ & $58 / \mathrm{M}$ & Unilateral & $\begin{array}{l}\text { Between the right } \\
\text { canine and the right } \\
\text { first molar }\end{array}$ & SG \\
\hline 55 & $\begin{array}{c}\text { Voss et al. }(2010) \\
{[65]}\end{array}$ & $50 / \mathrm{F}$ & Unilateral & Let canine & No biopsy \\
\hline 56 & $\begin{array}{l}\text { Dereci and Duran } \\
\text { (2012) [29] }\end{array}$ & $46 / \mathrm{M}$ & Unilateral & $\begin{array}{l}\text { Left canine and left } \\
\text { first premolar }\end{array}$ & No biopsy \\
\hline 57 & $\begin{array}{l}\text { Kim etal. (2014) } \\
{[8]}\end{array}$ & $44 / F$ & Bilateral & $\begin{array}{l}\text { Canine and premo- } \\
\text { lars in the right and } \\
\text { left mandible. }\end{array}$ & SG \\
\hline 58 & $\begin{array}{c}\text { Taysi et al. (2014) } \\
\text { [32] }\end{array}$ & $56 / \mathrm{M}$ & Unilateral & $\begin{array}{l}\text { Left canine and left } \\
\text { premolars }\end{array}$ & SG \\
\hline 59 & $\begin{array}{c}\text { Griffa et al. (2014) } \\
\text { [30] }\end{array}$ & $71 / \mathrm{M}$ & Unilateral & $\begin{array}{l}\text { Left lateral incisor to } \\
\text { left first premolar }\end{array}$ & SG \\
\hline
\end{tabular}

\begin{tabular}{|c|c|c|c|c|c|}
\hline Case & Authors(Year) & $\begin{array}{l}\text { Age/ } \\
\text { Sex }\end{array}$ & $\begin{array}{l}\text { Unilateral/ } \\
\text { Bilateral }\end{array}$ & Site & Content \\
\hline 60 & $\begin{array}{c}\text { Sekerciand } \\
\text { Sisman (2014) } \\
\text { [19] }\end{array}$ & $39 / \mathrm{M}$ & Bilateral & $\begin{array}{l}\text { Right canine-pre- } \\
\text { molar and left } \\
\text { incisor-premolar }\end{array}$ & No biopsy \\
\hline 61 & $\begin{array}{l}\text { Ozaki et al. (2015) } \\
\text { [12] }\end{array}$ & $76 / \mathrm{M}$ & $\begin{array}{l}\text { Simultaneous } \\
\text { anterior } \\
\text { unilateral and } \\
\text { posterior }\end{array}$ & $\begin{array}{l}\text { Anterior lesion at the } \\
\text { right first premolar }\end{array}$ & SG \\
\hline 62 & $\begin{array}{l}\text { Deyhimi et al. } \\
\text { (2016) [66] }\end{array}$ & $45 / \mathrm{M}$ & Unilateral & $\begin{array}{l}\text { Left lateral incisor } \\
\text { and left canine }\end{array}$ & $\mathrm{SG}, \mathrm{AT}, \mathrm{BV}$ \\
\hline 63 & $\begin{array}{l}\text { Present case } \\
\text { (2019) }\end{array}$ & $73 / \mathrm{M}$ & Unilateral & $\begin{array}{l}\text { Right lateral incisor } \\
\text { to right premolars } \\
\text { (edentulous) }\end{array}$ & CTBV \\
\hline
\end{tabular}

M: male; F: female; NM: Not mentioned; SG: salivary gland; AT: adipose tissue; CT: connective tissue; BF: bony fragments; BV: blood vessels

\section{CONCLUSION}

According to the clinical, imaginological and pathological findings, the current case suggests a Stafne's bone cavity (SBC) of uncommon localization and progression.

\section{REFERENCES}

1. Sisman Y,Miloglu 0, Sekerci AE, Yilmaz AB, Demirtas 0, Tokmak TT. Radiographic evaluation on prevalence of Stafne bone defect: a study from two centres in Turkey. Dentomaxillofac Radiol. 2012; 41(2):152-8.

2. Schneider T,Filo K, Locher MC, Gander T, Metzler P, GrätzKW etal. Stafne bone cavities: systematic algorithm for diagnosis derived from retrospective data over a 5-year period. Br J Oral Maxillofac Surg. 2014;52(4):369-74.

3. Philipsen HP, Takata T, ReichartPA, Sato S, Suei Y.Lingual and buccal mandibular bone depressions: a review based on 583 cases from a worldwide literature survey, including 69 new cases from Japan. Dentomaxillofac Radiol. 2002; 31(5):281-90.

4. Quesada-Gómez C, Valmaseda-CastellónE,Berini-Aytés L, Gay-EscodaC. Stafne bone cavity: a retrospective study of 11cases. Med Oral Patol Oral Cir Bucal. 2006; 11(3):E277-280

5. Turkoglu K, Orhan K. Stafne bone cavity in the anterior mandible.J Craniofac Surg. 2010; 21(6):1769-75.

6. Herbozo Silva P,Briones Sindermann D, Martinez Rondanell B. Giant mandibular bone defect: report of a case. J Oral Maxillofac Surg.2006; 64(1):145-50.

7. Smith MH, Brooks SL, Eldevik OP,Helman Jl. Anterior mandibular lingual salivary gland defect: a report of a case diagnosed with cone-beam computed tomography and magnetic resonance imaging. Oral Surg Oral Med Oral Pathol Oral Radiol Endod. 2007; 103(5):e71-78.

8. Kim H,Seok JY,Lee S, An J, Kim NR, Chung DH etal. Bilateral stafne bone cavity in the anterior mandible with heterotopic salivary gland tissue: a case report. Korean JPathol. 2014;48(3):248-9.

9. Herranz-Aparicio J, Figueiredo R, Gay-Escoda C. Stafne's bone cavity:An unusual case with involvement of the buccal and lingual mandibular plates. $J$ Clin Exp Dent. 2014;6(1):e96-99. 
10. Campos PS, Panella J, Crusoé-Rebello IM, Azevedo RA, Pena N, Cunha T. Mandibular ramus-related Stafne's bone cavity. Dentomaxillofac Radiol. 2004; 33(1):63-6.

11. Lee KH, Thiruchelvam JK, McDermottP.An Unusual Presentation of Stafne Bone Cyst. J Maxillofac Oral Surg. 2015; 14(3):841-4.

12. Ozaki H, Ishikawa S, Kitabatake K, Yusa K, Tachibana H, lino M. A Case of Simultaneous Unilateral Anterior and Posterior Stafne Bone Defects. Case Rep Dent. 2015;2015:983956.

13. Boffano P,Gallesio C, Daniele D, RocciaF. An unusual trilobate Stafne bone cavity. Surg Radiol Anat. 2013;35(4):351-3.

14. Friedrich RE, Zustin J, Scheuer HA, Assaf AT, Gröbe A. An unilateral basal bone defect of the mandible occupied by fatty tissue:Stafne's cavity. In Vivo. 2012; 26(6):1045-8.

15. Shimizu M, Osa N, Okamura K, Yoshiura K. CT analysis of the Stafne's bone defects of the mandible. Dentomaxillofac Radiol. 2006; 35(2):95-102

16. Sisman Y, Etöz OA, Mavili E, Sahman H, Tarim EE. Anterior Stafne bone defect mimicking a residual cyst: a case report. Dentomaxillofac Radiol. 2010;39(2):124-6.

17. Grellner TJ, Frost DE, Brannon RB. Lingual mandibular bone defect: report of three cases. J Oral Maxillofac Surg.1990;48(3):288-96.

18. Queiroz LM, Roch R.S, de Medeiros KB, da Silveira EJ, Lins RD. Anterior bilatera presentation of Stafne defect: an unusual case report. J Oral Maxillofac Surg. 2004;62(5):613-5.

19. Sekerci AE, Sisman Y.Bilateral anterior Stafne bone defect mimicking radicular cyst: report of a rare case with a review of the literature. Oral Radiology.2014; 30(1):115-22.

20. CamilleriG. Salivary gland inclusion in anterior mandible. Br Dent J.1963;114:515-6.

21. Friedman J. Ectopic sublingual glands: Report of a case. Oral Surg Oral Med Oral Pathol.1964:18(2):219-22

22. Miller AS, Winnick M. Salivary gland inclusion in the anterior mandible. Report of a case with a review of the literature on aberrant salivary gland tissue and neoplasms. Oral Surg Oral Med Oral Pathol. 1971;31(6):790-7.

23. Mizuno A, Kawabata T,Nakano Y,Motegi K. Lingual mandibular bone defectidiopathic bone cavity. Report of a case. Int J Oral Surg.1983;12(1):64-68.

24. ECS. Bone cavities situated near the angle ofthe mandible.J Am Dent Assoc. 1942;29:1969-1972.

25. Flores Campos PS, Carvalho Oliveira JA, Dantas JA, de Melo DP,Pena N, Santos LAN etal.Stafne Defect with Buccal Cortical Expansion: A Case Report. IntJ Dent 2010;2010:3.

26. Connor MS. Anterior lingual mandibular bone concavity. Report of a case. Oral Surg Oral Med Oral Pathol. 1979;48(5):413-4

27. Bergenholtz A,Persson G. Idiopathic bone cavities. A report of four cases. Oral Surg Oral Med Oral Pathol. 1963;16:703-12.

28. Buchner A, Carpenter WM, Merrell PW, Leider AS. Anterior lingual mandibular salivary gland defect. Evaluation of twenty-four cases. Oral Surg Oral Med Ora Pathol. 1991;71(2):131-6.

29. Dereci 0, Duran, S. Intraorally exposed anterior Stafne bone defect: a case report. Oral Surg Oral Med Oral Pathol Oral Radiol. 2012; 113(5):e1-3.

30. Griffa A,ZavatteroE, Passalacqua F, Berrone S. Anterior Stafne bone defect mimicking an odontogenic cyst. J Craniofac Surg. 2014;25(3):1126-8.

31. Miloğlu Ö, Sekerci AE, Yasa Y, Dagistan S. Unilateral bone cavities situated near the angle of the mandibula. JCraniofac Surg. 2015;26(1):e27-28.
32. Taysi M, Ozden C,Cankaya B, Olgac V, YıldırımS. Stafne bone defect in the anterior mandible. Dentomaxillofac Radiol. 2014;43(7):20140075.

33. Krafft T,Eggert J,Karl MA. Stafne bone defect in the anterior mandible--a diagnostic dilemma. Quintessence Int. 2010;41(5):391-3.

34. Venkatesh E. Stafne bone cavity and cone-beam computed tomography:a report of two cases. J Korean Assoc Oral Maxillofac Surg. 2015;41(3):145-8.

35. Richard EL, Ziskind J. Aberrant salivary gland tissue in mandible. Oral Surg Oral Med Oral Pathol. 1957;10(10):1086-90.

36. Araiche M, Brode H. Aberrant salivary gland tissue in mandible. Oral Surg Ora Med Oral Pathol. 1959;12(6):727-9.

37. Palladino VS, Rose SA, Curran T. Salivary gland tissue in the mandible and stafne's mandibular 'cysts'. J Am Dent Assoc. 1965;70:388-93.

38. Abramson AS: Ectopic submaxillary gland in the mandible: report of case. $J$ AmDent Assoc. 1966:73(5):1114-6.

39. Malkin M,Berg R. Stafne defect of the anterior mandible. NY State DentJ. $1974 ; 40(1): 17-9$.

40. Forrest WR. Ectopic salivary gland. Oral Surg Oral Med Oral Pathol.1974; 37(4):653-4.

41. Stene T,Pedersen KN. Aberrant salivary gland tissue in the anterior mandible. Oral Sur Oral Med Oral Pathol. 1977;44(1):72-75.

42. Plezia RA. Unusual static bone cavity. Oral Surg Oral Med Oral Pathol. 1977:43(6):979

43. Sandy JR, Williams DM. Anterior salivary gland inclusion in the mandible: Pathological entity or anatomical variant?. Br J Oral Surg. 1981;19(3):223-9.

44. Layne EL, Morgan AF, Morton TH. Anterior lingual mandibular bone concavity: report of case. J Oral Surg. 1981;39(8):599-600.

45. Gosney MB, Giles AD. Atypical mandibular salivary inclusion. Br Dent J. 1981;151(11):381-2.

46. Hayashi Y,Kimura Y,Nagumo M. Anterior lingual mandibular bone concavity. Report of a case. Oral Surg Oral Med Oral Pathol.1984;57(2):139-42.

47. Miller AS, Fantasia JE. Mandibular radiolucency I. Gen Dent.1986;34(6):491-5.

48. Ström C, Fiellström CA. An unusual case of lingual mandibular depression. Oral Surg Oral Med Oral Pathol. 1987;64(2):159-61.

49. Anneroth G, Berglund G, Kahnberg KE. Intraosseous salivary gland tissue of the mandible mimicking a periapical lesion. Int J Oral Maxillofac Surg. 1990;19 (2):74-5

50. Tominaga K, Kuga Y, Kubota K, Ohba T.Stafne's bone cavity in the anterior mandible: report of a case. Dentomaxillofac Radiol. 1990;19(1):28-30.

51. ChildersEL, Johnson JD, Warnock GR, KratochvilFJ. Asymptomatic periapical radiolucent lesion found in an area of previous trauma. J Am Dent Assoc. 1990;121(6):759-60.

52. Salman L, Chaudhry AP.Malposed sublingual gland in the anterior mandible: variant of Stafne's idiopathic bone cavity. Compendium. 1991;12(1):40,42-3.

53. Barak S, Katz J, Mintz S. Anterior lingual mandibular salivary gland defect--a dilemma in diagnosis. Br J Oral Maxillofac Surg. 1993;31(5):318-20.

54. Thomas LP,Abramovitch K. Case report. Diagnosis and management of an anterior lingual mandibular salivary gland defect. Dentomaxillofac Radiol. 1996; 25(4):207-10

55. Apruzzese D, Longoni S. Stafne cyst in an anterior location. J Oral Maxillofac Surg. 1999;57(3):333-8. 
56. Katz J,Chaushu G, Rotstein I.Stafne's bone cavity in the anterior mandible: a possible diagnostic challenge.J Endod. 2001;27(4):304-7.

57. de Courten A, Küffer R, Samson J,Lombardi T. Anterior lingual mandibular salivary gland defect (Stafne defect) presenting as a residual cyst. Oral Surg Oral Med Oral Pathol Oral Radiol Endod. 2002;94(4):460-4.

58. Dorman M,Pierse D. Ectopic salivary gland tissue in the anterior mandible: a case report. Br Dent J. 2002;193(10):571-72.

59. Phillips A, Yates C. Case report: anterior lingual mandibular cortical bone concavity. Dent Update. 2004;31(3):175-6.

60. Belmonte-Caro R, Velez-Gutierrez MJ, Garcia De la Vega-Sosa FJ, Garcia-PerlaGarcia A, Infante-Cossio PA, Diaz-Fernandez JM etal. A Stafne's cavity with unusual location in the mandibular anterior area. Med Oral Patol Oral Cir Bucal. 2005;10(2):173-9.

61. Solomon LW, Pantera EA, Monaco E, White SC, Suresh L. A diagnostic challenge: anterior variant of mandibular lingual bone depression. Gen Dent. 2006;54(5):336-40.
62. Nah KS, Jung YH, Cho BH. Unusual stafne bone cavity mimicking infected cyst or neural origin tumor. Korean Journal of Oral and Maxillofacial Radiology. 2007;37(4):221-3.

63. Bornstein MM, Wiest R, Balsiger R, ReichartPA. Anterior Stafne's bone cavity mimicking a periapical lesion of endodontic origin: report of two cases. $J$ Endod. 2009;35(11):1598-1602

64. Florin W,Mandel L.Stafne's bone cavity anterior mandible. Case report.NY Stat. Dent J. 2010; 76(3):36-38.

65. Voss PJ,Metzger MC, Schulze D, Loeffelbein DJ, Pautke C, Hohlweg-MajertB. Anterior lingual mandibular bone cavity as a diagnostic challenge: two case reports. J Oral Maxillofac Surg. 2010;68(1):201-4.

66. Deyhimi P,Darisavi S, Khalesi S. Stafne bone cavity with ectopic salivary gland tissue in the anterior of mandible.Dent Res J. 2016;13(5):454-7.

\section{Karen Pintado-Palomino}

(Corresponding address)

Postgraduate Program in Oral Rehabilitation -

Universidad Nacional Mayor de San Marcos, Lima - Perú.

Date submitted: 2019 Aug 03

E-mail: karpintado@gmail.com 